\title{
SIMULATION OF FIBRE REINFORCED CONCRETE STRUCTURAL FAILURE BASED ON BENDING TEST RESULTS
}

\author{
Ulvis Skadins, Mareks Bokta \\ Latvia University of Life Sciences and Technologies, Latvia \\ ulvis.skadins@1lu.lv,mareks.bokta@gmail.com
}

\begin{abstract}
Composite materials like steel fibre reinforced concrete (SFRC) gain attention among engineers and designers. It is possible by adding fibres to increase ductility of such brittle materials as concrete. However, such materials are difficult to design, because the mechanical properties of SFRC are hard to predict. Nonlinear computer modelling based on the Finite Element Analysis (FEA) can be utilised to predict failure of a composite structure, if the material properties are defined correctly. Material model describing properties in tension and compression of the composite need to be defined. In practice uniaxial tension tests in case of SFRC are difficult to perform, therefore the properties of the material are represented by standard laboratory bending tests. These results cannot be used directly in nonlinear FEA simulation software. The aim of the study is to find the link between the results of standard bending tests and the material model in tension that could be used in simulation software. In the study ATENA GiD software is used for simulation purposes. The simulation is performed in conjunction with experimental results of 24 SFRC beams, tested according to the standard EN 14651. The material model of SFRC is obtained by an improved inverse analysis based on the bending test results. Parametric analysis of the SFRC material model is performed. As a result, a numerical method for converting the bending test results to the material model in tension is suggested. The evaluation of the proposed model shows $\pm 2.5 \%$ error at $3.5 \mathrm{~mm}$ of crack mouth opening distance in average, if the predicted and the actual fracture energy is compared.
\end{abstract}

Keywords: fibre reinforced concrete, tension function, inverse analysis, nonlinear finite element analysis.

\section{Introduction}

By adding short steel fibres to concrete less than $1.0 \%$ by volume, the strength properties of the material do not change a lot. However, the concrete becomes more ductile. This property allows structural engineers to replace the conventional reinforced concrete with the less labour-consuming steel fibre reinforced concrete (SFRC) in certain types of structures. To evaluate the load bearing capacity of SFRC structures, the structural designer needs an appropriate software and information about the material properties.

To model a SFRC structure in different loading stages a nonlinear finite element analysis (FEA) software is strongly recommended. The software must be capable of handling the nonlinear material properties and possible strain softening behaviour in tension. If the correct material properties, especially tension function, are defined, the software opens huge possibilities for structural designers [1].

In case of ordinary SFRC the tensile properties can be determined by laboratory tests according to the standard EN 14651 [2]. According to the standard notched SFRC prisms are tested in three-point bending and the applied load versus the crack mouth opening displacement (CMOD) is recorded. The test gives information about the material's tensile behaviour in bending, but it cannot be used to describe the behaviour in pure tension, because in case of SFRC it is different $[1 ; 3]$. Therefore, the test results are not valid to define the material properties in the FEA software. On the other hand, the laboratory tests for SFRC in direct tension are rather complicated, thus not supported by most of the labs.

To address this issue different methods of inverse analysis are used. In the analysis the material properties in tension are determined by manual $[4 ; 5]$ or numerical $[6 ; 7]$ iteration so that $F-C M O D$ (or load-deflection) behaviour of a theoretical model would fit to the experimental one. The manual approach is comparatively a labour-consuming process, because the position of the points defining the tension function are to be guessed. Sajdlova et al [8] suggested to start with the simple definition proposed by RILEM to be modified by the iteration process. Although there are some guidelines available, the prediction of the number and the position of the points in both directions takes time and experience.

The numerical method proposed by Roelfstra et al [6] is based on the bilinear approximation of concrete strain softening behaviour. The right crack opening law for a specific material is found by solving an optimisation task using numerous computer iterations. There are variety of other numerical methods used for the inverse analysis, including a use of a multi-criteria analysis that is used to make decisions for identification of SFRC properties [7]. Novak and Lehky [9] proposed a concept to use 
artificial neural network in conjunction with the inverse analysis. Besides the approaches mentioned so far, methods based on fracture energy are suggested [10].

The aim of this paper is to share the authors' experience to ease the process of determining the tension properties of SFRC to be used in nonlinear FEA software. Two methods are developed. The first is a slightly improved manual inverse analysis. The second is a mathematical model to determine the tension function by numerical calculations based on the standard bending test results.

\section{Materials and methods}

\subsection{Experimental laboratory tests}

The experimental results are obtained by testing 24 notched beam specimens in flexure according to the standard EN 14651 [2]. All the specimens can be grouped in two samples, S2 and S3, with two different amounts of fibres: 30 and $60 \mathrm{~kg} \cdot \mathrm{m}^{-3}$, respectively. In all cases fibres with hooked ends, $50 \mathrm{~mm}$ length, and $0.75 \mathrm{~mm}$ diameter (KAMPE HAREX, DE 50/0.75 N) were used. The specimens were manufactured by company "MB Betons". Self-compacting concrete with the consistency class S5 was used. The concrete mean cylindric compressive strength on the day of CMOD tests of the prisms was $35.65 \mathrm{MPa}$ (CoV 0.032) for sample S2 and $34.47 \mathrm{MPa}(\mathrm{CoV} 0.033)$ for sample S3. The F-CMOD curves for the prisms are given in Fig. 1. Based on the curves, the flexural tensile strength and mean tensile strength are calculated. The measured and calculated strength and geometrical properties for each prism are given in Table 1.

Table 1

Geometrical and strength properties of the tested prisms

\begin{tabular}{|c|c|c|c|c|c|c|c|}
\hline Sample & Specimen & $\begin{array}{l}b, \\
\mathbf{m m}\end{array}$ & $\begin{array}{l}h_{s p}, \\
\text { mm }\end{array}$ & $\begin{array}{l}F_{L}, \\
\mathbf{k N}\end{array}$ & $\begin{array}{c}C M O D_{L}, \\
\mathrm{~mm}\end{array}$ & $\begin{array}{l}f_{c t, L}, \\
\text { MPa }\end{array}$ & $\begin{array}{l}f_{c t m}, \\
\text { MPa }\end{array}$ \\
\hline \multirow[t]{7}{*}{ S2 } & 1 & 153.95 & 123.835 & 13.46 & 0.04 & 4.276 & 2.897 \\
\hline & 2 & 154.215 & 123.965 & 14.01 & 0.04 & 4.434 & 3.004 \\
\hline & 3 & 153.45 & 123.115 & 14.37 & 0.04 & 4.634 & 3.138 \\
\hline & 4 & 151.93 & 123.675 & 13.23 & 0.04 & 4.27 & 2.892 \\
\hline & 5 & 152.39 & 123.885 & 13.96 & 0.04 & 4.477 & 3.033 \\
\hline & 6 & 153.995 & 124.32 & 13.59 & 0.04 & 4.282 & 2.902 \\
\hline & 7 & 151.27 & 123.525 & 15.59 & 0.04 & 5.066 & 3.431 \\
\hline \multirow[t]{5}{*}{$\overline{S 2}$} & 8 & 154.00 & 123.745 & 13.56 & 0.04 & 4.313 & 2.922 \\
\hline & 9 & 153.95 & 123.665 & 14.13 & 0.04 & 4.501 & 3.049 \\
\hline & 10 & 153.855 & 123.410 & 13.38 & 0.03 & 4.283 & 2.901 \\
\hline & 11 & 154.24 & 123.300 & 14.3 & 0.04 & 4.574 & 3.097 \\
\hline & 12 & 153.425 & 123.625 & 13.77 & 0.04 & 4.404 & 2.983 \\
\hline \multirow[t]{12}{*}{ S3 } & $1 *$ & 152.58 & 124.140 & - & - & - & - \\
\hline & 2 & 154.22 & 123.790 & 13.03 & 0.04 & 4.135 & 2.801 \\
\hline & 3 & 151.655 & 123.940 & 12.89 & 0.03 & 4.15 & 2.812 \\
\hline & 4 & 152.175 & 123.860 & 12.83 & 0.04 & 4.122 & 2.792 \\
\hline & 5 & 151.9 & 123.635 & 12.33 & 0.04 & 3.983 & 2.698 \\
\hline & 6 & 150.945 & 124.070 & 14.09 & 0.04 & 4.548 & 3.081 \\
\hline & 7 & 151.74 & 124.225 & 13.08 & 0.04 & 4.189 & 2.839 \\
\hline & 8 & 151.82 & 124.525 & 13.3 & 0.04 & 4.237 & 2.872 \\
\hline & 9 & 151.675 & 124.045 & 14.52 & 0.04 & 4.666 & 3.161 \\
\hline & 10 & 150.715 & 124.335 & 12.03 & 0.03 & 3.872 & 2.624 \\
\hline & 11 & 154.04 & 123.400 & 12.02 & 0.03 & 3.843 & 2.603 \\
\hline & 12 & 150.725 & 123.985 & 12.46 & 0.04 & 4.033 & 2.732 \\
\hline
\end{tabular}

* rejected due to broken CMOD measuring device 
The peak load $F_{L}$ is determined as the maximum force developed within the first $0.05 \mathrm{~mm}$ of the crack mouth opening displacement. The flexural tensile strength $f_{c t, L}$ is determined by equation (1) [2]:

$$
f_{c t, L}=\frac{3 \cdot F_{L} \cdot l}{2 \cdot b \cdot h_{s p}^{2}}
$$

where $l$-span of the beam;

$b$ - width of the cross-section;

$h_{s p}$ - distance from the top of the beam to the tip of the notch.

The mean tensile strength $f_{c t m}$ is derived from the formula given in EC2 [11] as follows:

$$
f_{c t m}=\frac{f_{c t, L}}{1.6-h_{s p} / 1000},
$$
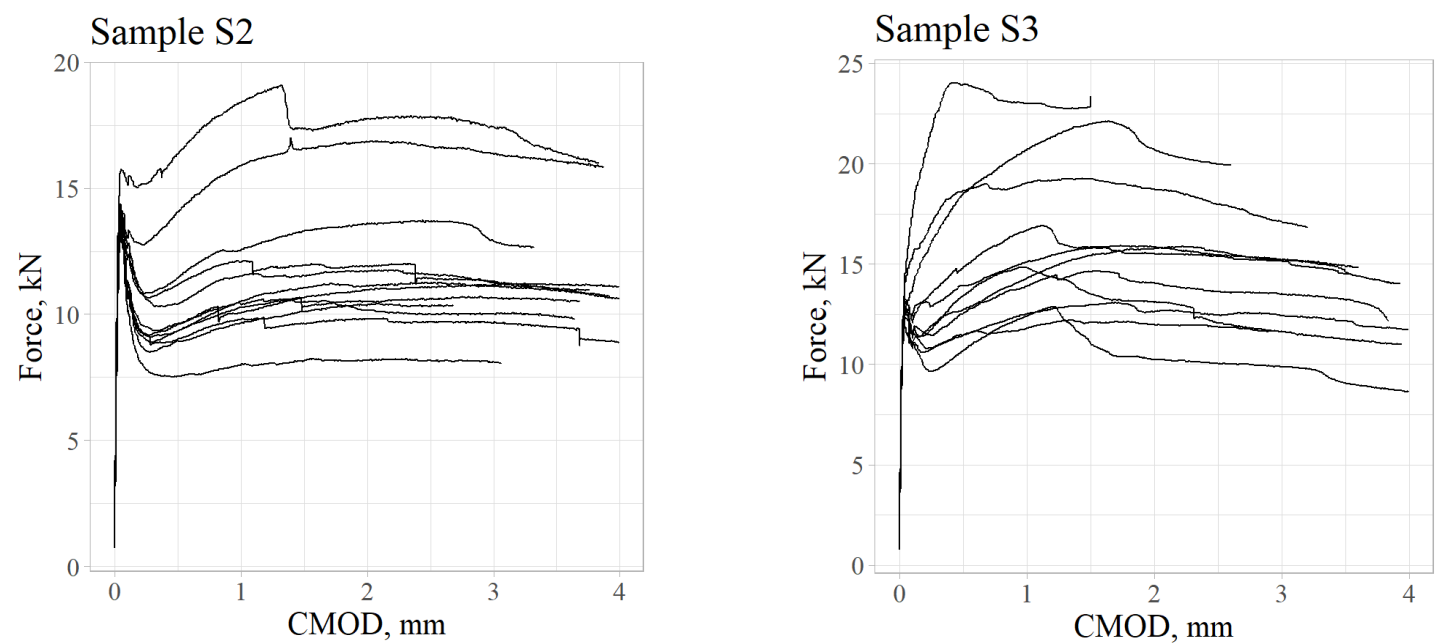

Fig. 1. F-CMOD curves for prisms tested in the laboratory

\subsection{Manual inverse analysis}

The inverse analysis is performed partly based on the procedure described in the ATENA Program Documentation [4]. To obtain the tension function, the results from the experimental flexure tests are used. The specimen and the test setup used in the experiments are modelled in the ATENA GiD software (see Fig. 2). The material properties are defined according to the tested properties given in section 2.1. Other properties for compressive behaviour are modified according to the suggestions by the ATENA documentation [4]. The tensile strength is taken as the mean tensile strength $f_{c t m}$ according to Table 1 , determined by the equations (1) and (2). Typical crack propagation and failure mode of the theoretical model (see Fig. 3) correspond well with the experimental beams.

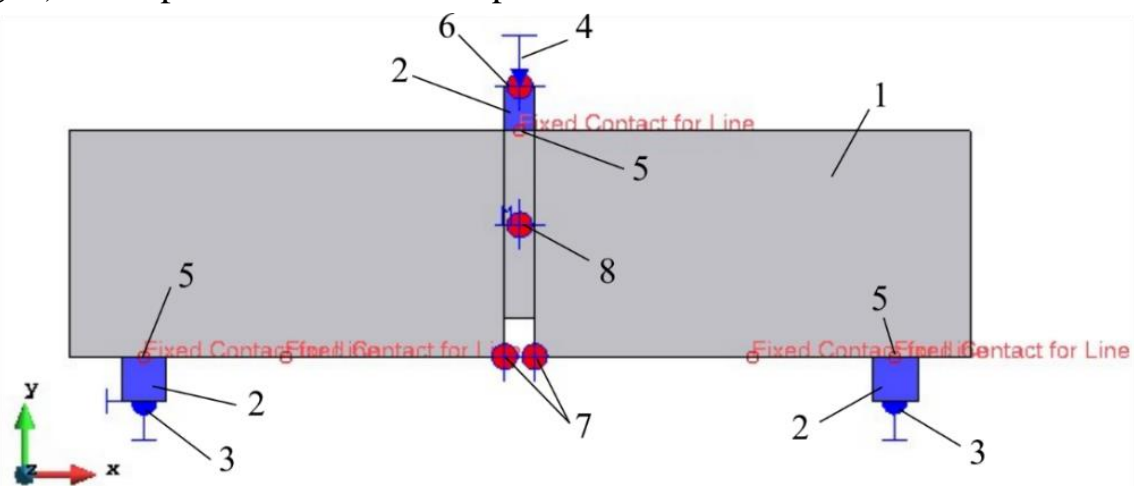

Fig. 2. Model of the prism defined in ATENA GiD: 1 - surface element with user defined material properties for SFRC beam; 2 - surface elements modelled as supports with linear-elastic material properties; 3 - constraints for points; 4 - applied displacement in $y$ direction; 5 - fixed contacts between two surfaces; 6 - monitor points for reaction; 7 - monitor points for displacements in $x$ direction; 8 - monitor point for the maximum crack width in the surface 


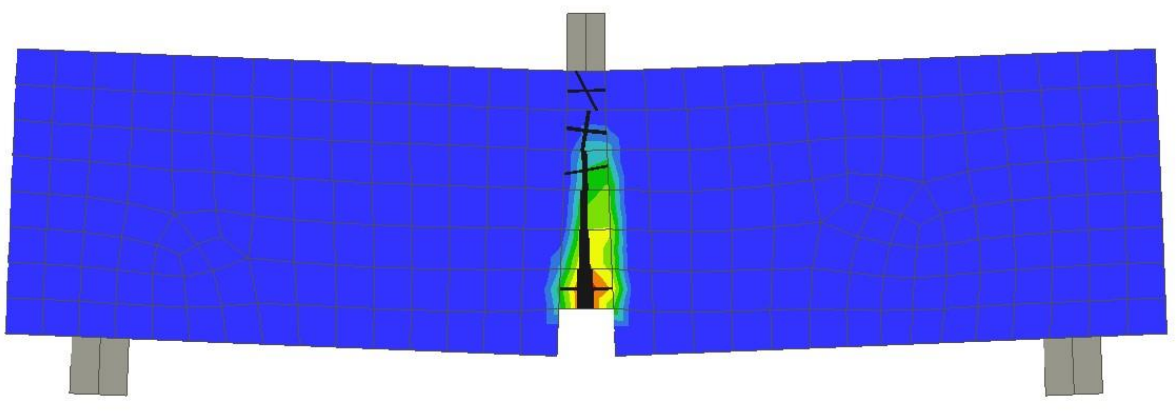

Crack Width

cod1

[m]

0.000430

0.000376

0.000322

0.000269

0.000215

0.000161

0.000107

0.000054

0.000000

Deformation scale

20

Fig. 3. Crack propagation in the specimens simulated by ATENA

To find the appropriate tension function, the following approach is developed. A series of fixed COMD values are chosen. For each given CMOD a tension function value is adjusted. For the tension value the recorded $F-C M O D$ data of the tested SFRC beams expressed as the force ratio $r_{F}$ are used as the initial values multiplied by the transition coefficient $k$. The force ratio $r_{F}$ at every given CMOD value is calculated by dividing the measured force $F_{i}$ with the force at the limit of proportionality (LOP) $F_{L}$ :

$$
r_{F}=\frac{F_{i}}{F_{L}},
$$

where $F_{i}$ - determined as the average force in the distance $a$ (see Fig. 4(b)) next to the given CMOD.

In this study the following $C M O D$ values (in $\mathrm{mm}$ ) were used:

$$
\overrightarrow{C M O D}=\{0,0.05,0.1,0.2,0.5,1.0,1.5,2.0,2.5,3.0,3.5,20\}+C M O D_{F_{L}},
$$

where $C M O D_{F_{L}}-$ crack mouth opening displacement at LOP.

The $C M O D=20 \mathrm{~mm}$ is considered as the ultimate crack opening distance that can be related to the length of the fibres. It is assumed here that the $C M O D$ cannot exceed 40 to $50 \%$ of the fibre length, thus $0.4 \cdot 50 \mathrm{~mm}=20 \mathrm{~mm}$ as the ultimate $C M O D$ value was used. The length of the distance $a$ for each CMOD point is taken as:

$$
a_{i}=\min \left\{\begin{array}{c}
C M O D_{i}-C M O D_{i-1}, \\
0.2 \mathrm{~mm}
\end{array}\right.
$$

The transition from the force ratio $r_{F}$ to the tension function is performed by means of the coefficient $k$ :

$$
r_{F . t}=k \cdot r_{F},
$$

where $r_{F . t}-$ force ratio for the tension function.

At $r_{F . t}=1.0$ the tensile strength corresponds to the LOP. Conversion from CMOD to strains is needed for the FEA software and it is done as follows:

$$
\varepsilon_{i}=\left(C M O D_{i}-C M O D_{F_{L}}\right) / l_{c h},
$$

where $l_{c h}$-characteristic length, which is taken equal to the size of finite elements at the location, where cracks develop. In this study the size of the finite element is $20 \mathrm{~mm}$.

The sizes of the finite elements of the model are kept constant in the area of the possible fracture zone.

The vertical displacement is applied by steps of $0.02 \mathrm{~mm}$. The CMOD is calculated as the difference of the two horizontal measurements at each side of the notch. As a result, a theoretical $F-C M O D$ curve is obtained and compared with the experimental one. Afterwards the values of the transition coefficients are adjusted and the ATENA calculation is performed. The procedure is repeated until the match between the theoretical and experimental $F-C M O D$ curves of the given specimen is satisfactory. The 
theoretical curves and the transition coefficients were obtained for 6 specimens of sample S2. Correlation between the coefficient values and other parameters was analysed.

\subsection{Numerical model}

Based on the results obtained from manual inverse analysis (see section 2.2.), a numerical approach was developed. The aim of this approach is to define a model to calculate the transition coefficients $k$ and the tension function to avoid the manual iteration process. The developed model is illustrated in Fig. 4.
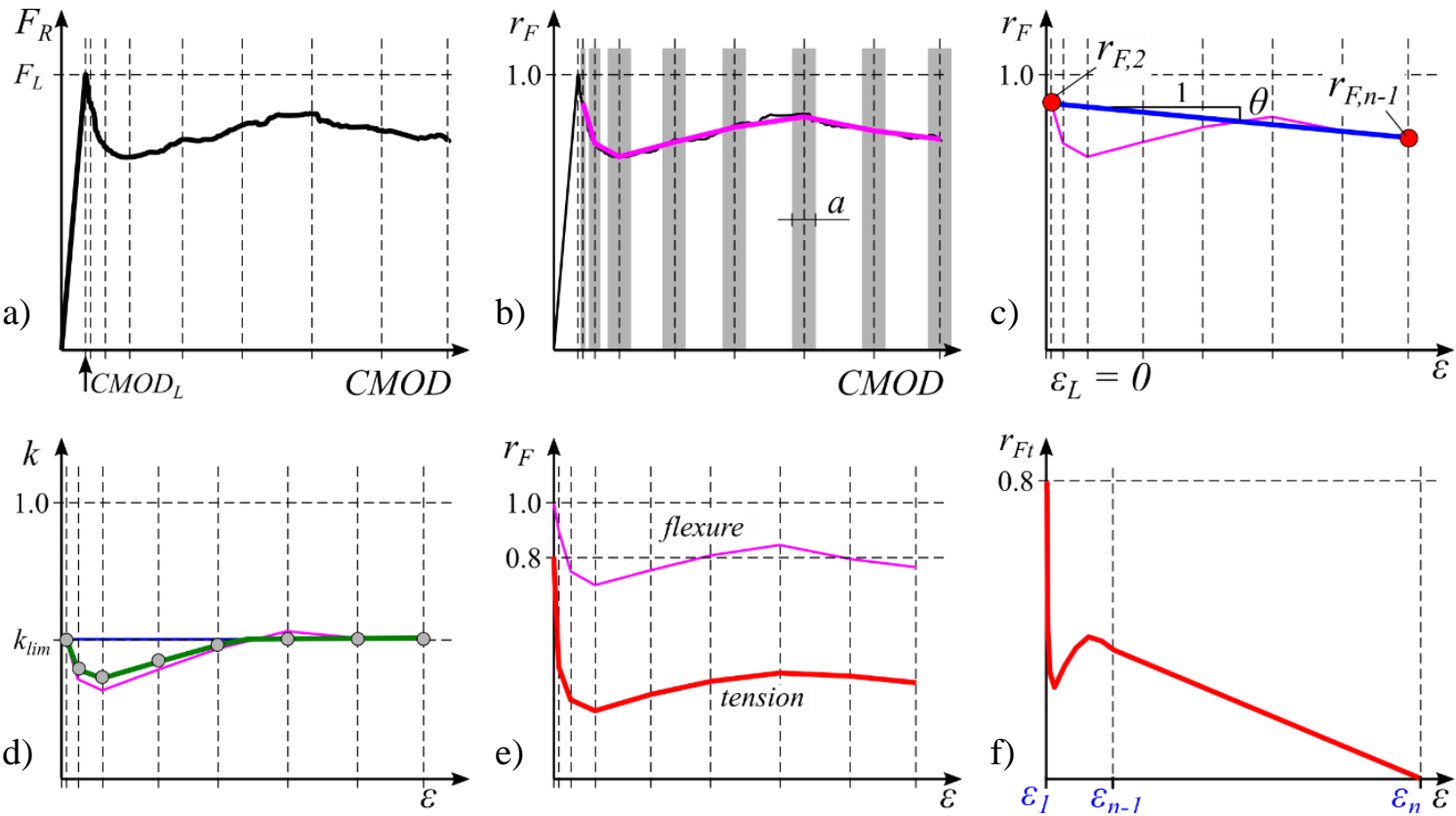

Fig. 4. Proposed method for obtaining the tension function: a - experimental F-CMOD curve divided into intervals; $\mathrm{b}$ - experimental curve expressed in terms of the force ratio $r_{F}$ and approximated (magenta); $\mathrm{c}$ - approximated curve (magenta) converted to strains with a line (blue) connecting two characteristic points (red); $\mathrm{d}$ - transition coefficient values (green line); $\mathrm{e}$ - transition from the approximated flexure curve (magenta) to the tension function (red); $\mathrm{f}$ - full representation of the tension function as defined in ATENA software

The first two steps are shown in Fig. 4(a) and (b) are described in section 2.2. After converting CMOD to strains $\varepsilon$ according to equation (7), the slope coefficient $\theta$ between the points 2 and $n-1$ is calculated (see Fig. 4(c)):

$$
\theta=\frac{r_{F, 2}-r_{F, n-1}}{\varepsilon_{n-1}-\varepsilon_{2}}
$$

where $r_{F, 2}, r_{F, n-1}, \varepsilon_{2}, \varepsilon_{n-1}$ - force ratios and strains corresponding to the elements 2 , and $n-1$ in $\overrightarrow{\mathrm{CMOD}}$ vector (4), respectively;

$n$ is the number of elements in the vector.

The points 2 and $n-1$ are taken because in this range the tension function needs to be defined. The values for the first and the last point are fixed and do not require any calculation.

Using the slope coefficient $\theta$, the values of the force ratio $r_{F, i}$ are converted to the values of the coefficient $k_{i}^{*}$, so that the line connecting the point 2 and $n-1$ is horizontal:

$$
k_{i}^{*}=r_{F, i}-\left(\left|\varepsilon_{n-1} \cdot \theta\right|-\varepsilon_{i} \cdot \theta\right), i=2, \cdots, n-1
$$

The values of the transition coefficient $k$ at each point are got by moving the $k^{*}$ polyline so that $k_{2}^{*}$ and $k_{n-1}^{*}$ values are equal to $k_{\text {lim }}$ (magenta line in Fig. 4(d)). The final $k$ values are taken smaller or equal to $k_{\text {lim }}$ as it is represented in equation (10) and by the green polyline and grey dots in Fig. 4(d).

$$
k_{i}=\min \left\{\begin{array}{c}
k_{i}^{*} \cdot \beta-\left(k_{2} \cdot \beta+k_{\text {lim }}\right) \\
k_{\text {lim }}
\end{array},\right.
$$


where $k_{\text {lim }}=0.45-$ upper limit value of the coefficient $k$ after LOP;

$\beta=0.7$ - reduction coefficient.

The values of the first and the last element of the coefficient vector $k_{1}$ and $k_{n}$ are taken equal to 0.8 and 0 , respectively. The values mentioned in this paragraph are obtained from the results of the manual inverse analysis presented in the section 3 . The force ratio values for the tension function is then found by the equation (6) as shown by the red polyline in Fig. 4(e) and (f).

\section{Results and discussion}

Values of the transition coefficient $k$ for the analysed specimens are plotted in Fig. 5. It was found that the values of $k$ depend on two parameters: strain $\varepsilon$ and force ratio $r_{F}$. For higher $r_{F}$ values the $k$ values increase. However, it is true for small strain values. With the increase of the strains, the influence of $r_{F}$ decreases. The correlation between $k$ and $r_{F}$ for different $\varepsilon$ values is shown in Fig. 6 . If the coefficient at LOP, $k_{1}$, is taken equal to 0.8 , the coefficient at other strain values does not exceed 0.45 . In all the cases $k_{2}$ values are 0.45 , if it is set at the strain $\varepsilon=0.0025$, and the strain at the LOP is set to zero.

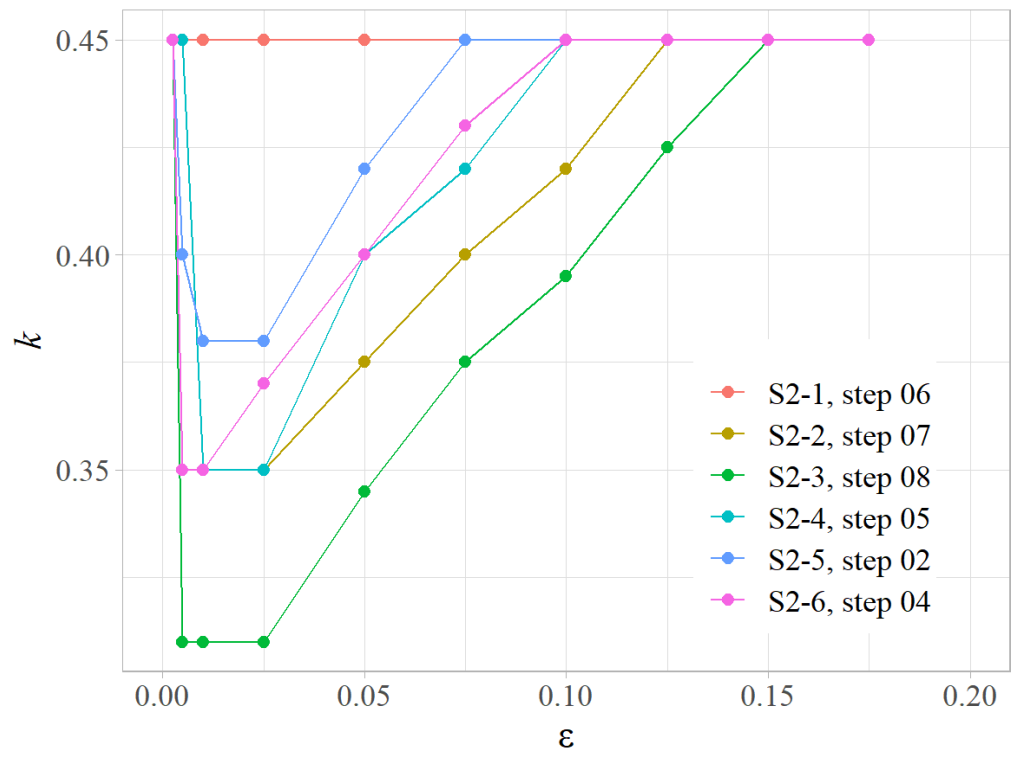

Fig. 5. Transition coefficient $\boldsymbol{k}$ obtained by manual inverse analysis

The experimental $F-C M O D$ curves (see Fig. 1) are compared with theoretical curves, which are obtained by ATENA Science software and the tension function properties determined by the manual inverse analysis (section 2.2.) and the proposed model (section 2.3.). The comparison for sample S2 given in Fig. 7 shows rather good compliance between the theoretical and experimental results. Both proposed methods lead to almost identical $F-C M O D$ curves. However, in case of sample S3, which due to the increased amount of fibres showed higher residual strength values, some discrepancies are revealed. For specimens S3-6, S3-8 and S3-9 a very steep increase of residual strength after LOP was observed. In these cases, the residual strength was underestimated for about 5 to $15 \%$.

The numerical comparison of the results is performed based on two parameters: the force and the area under the $F-C M O D$ curve (fracture energy) at a given CMOD value. The error is calculated by the following formula:

$$
\text { error }=(\text { Theor }-\operatorname{Exp}) / \operatorname{Exp} \times 100 \%,
$$

where Theor and Exp are the compared theoretical and experimental values, respectively.

The area $G$ under the $F-C M O D$ curve for each $\overrightarrow{C M O D}$ value is calculated by integration according to equation (12).

$$
G_{i}=\int_{0}^{C M O D_{i}} F(C M O D) d C M O D
$$


The estimated errors for the areas $G$ and the forces $F$ are represented by boxplots in Fig. 8, 9. The average error for $G$ is around $20 \%$ for the force at LOP $(C M O D=0.05 \mathrm{~mm})$, but the error reduces rapidly to around $2.5 \%$ for CMOD $>0.2 \mathrm{~mm}$. The results are similar for both samples, however, in the case of S3 they are more scattered and tend to slightly overestimate actual $G$ values. Kooiman [12], using the inverse analysis procedure, proposed by [6] set the limit for the error of fracture energy to be less than 10\%. In his studies the area under the curves till $20 \mathrm{~mm}$ for $30 \mathrm{~mm}$ fibres and till $70 \mathrm{~mm}$ for $60 \mathrm{~mm}$ fibres was compared. Sucharda [7] has not provided any numerical comparison for his results, but an approximate evaluation suggests that the error varies from around $10 \%$ at small displacements $(0.5 \ldots 1.0 \mathrm{~mm})$ to around $2 \%$ at $4 \mathrm{~mm}$ displacement. The data were acquired by a plot digitizer for the specimens with fibre amount of $1.0 \%$ (samples H10 and A10).
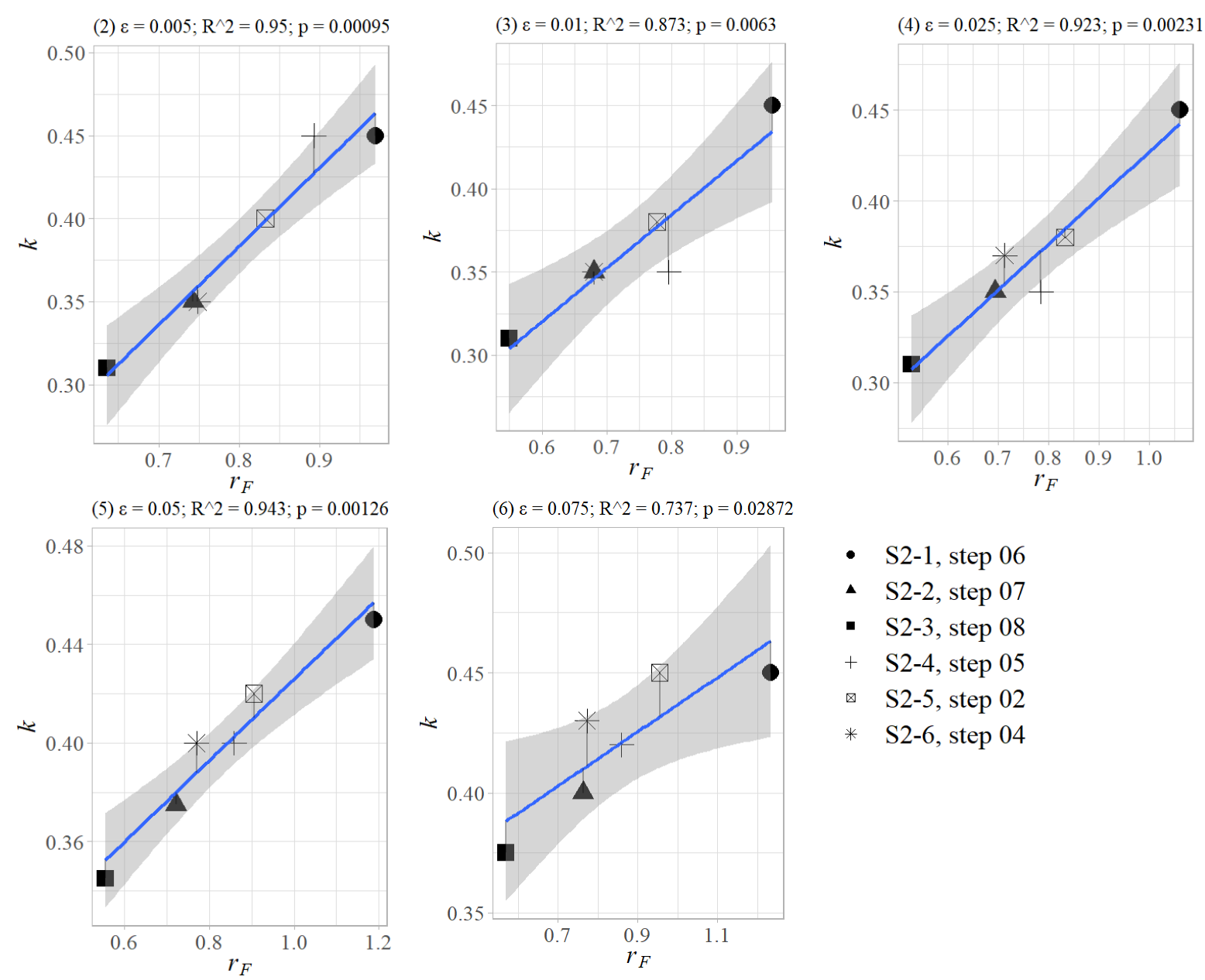

- S2-1, step 06

- S2-2, step 07

- $\quad$ S2-3, step 08

+ S2-4, step 05

$\bowtie \quad$ S2-5, step 02

* S2-6, step 04

Fig. 6. Correlation between $\boldsymbol{k}$ and $\boldsymbol{r}_{\boldsymbol{F}}$ at different $\varepsilon$ values

The errors of $F$ values do not show any pattern, but for most cases they lie between $-7.5 \%$ and $5 \%$ for sample S2 (fibres $30 \mathrm{~kg} \cdot \mathrm{m}^{-3}$ ), and between $-10 \%$ and $10 \%$ for sample S3 (fibres $60 \mathrm{~kg} \cdot \mathrm{m}^{-3}$ ). These deviations are relatively small comparing to the scatter of the experimental residual strength for specimens of the same sample (see Fig. 1), where the difference between the residual strength of two specimens can reach up to $\sim 100 \%$.

The advantage of the numerical approach proposed in this study is that the tension function can be determined without any iteration. In contrast, the methods proposed by other researchers require numerous iterations, thus using more computational time, or the accuracy of simulation is reduced. Kooiman points that the balance he has found for his approach is 500 iterations [12]. On the other hand, the proposed method has to be validated for other types of fibre reinforced cement composites. Special attention needs to be paid to the materials having distinctive strain hardening behaviour at small crack opening distances. 

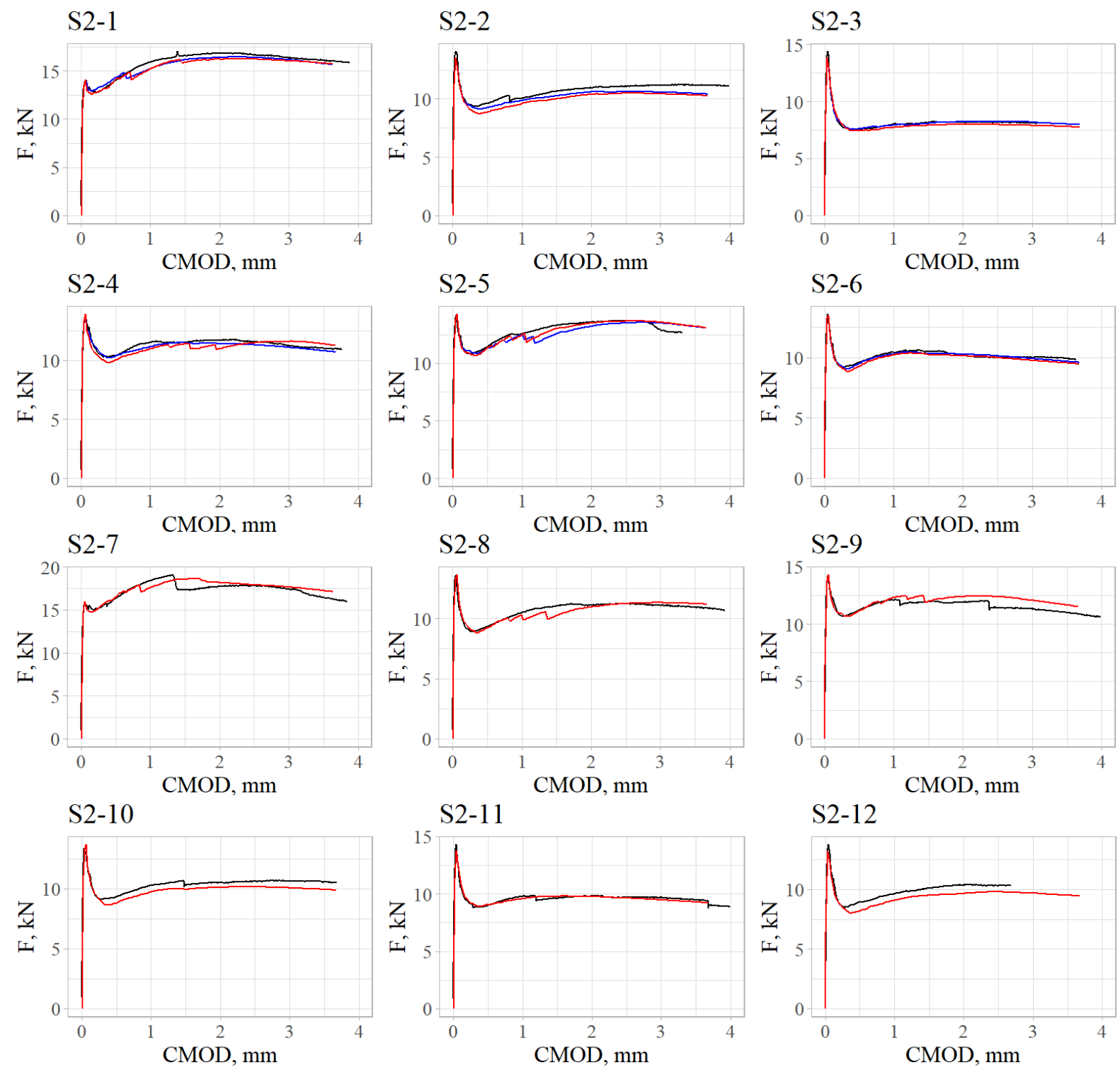

Fig. 7. Comparison between the experimental (black) and the ATENA results based on the tension function from the manual inverse analysis (blue) and from the proposed numerical model (red)
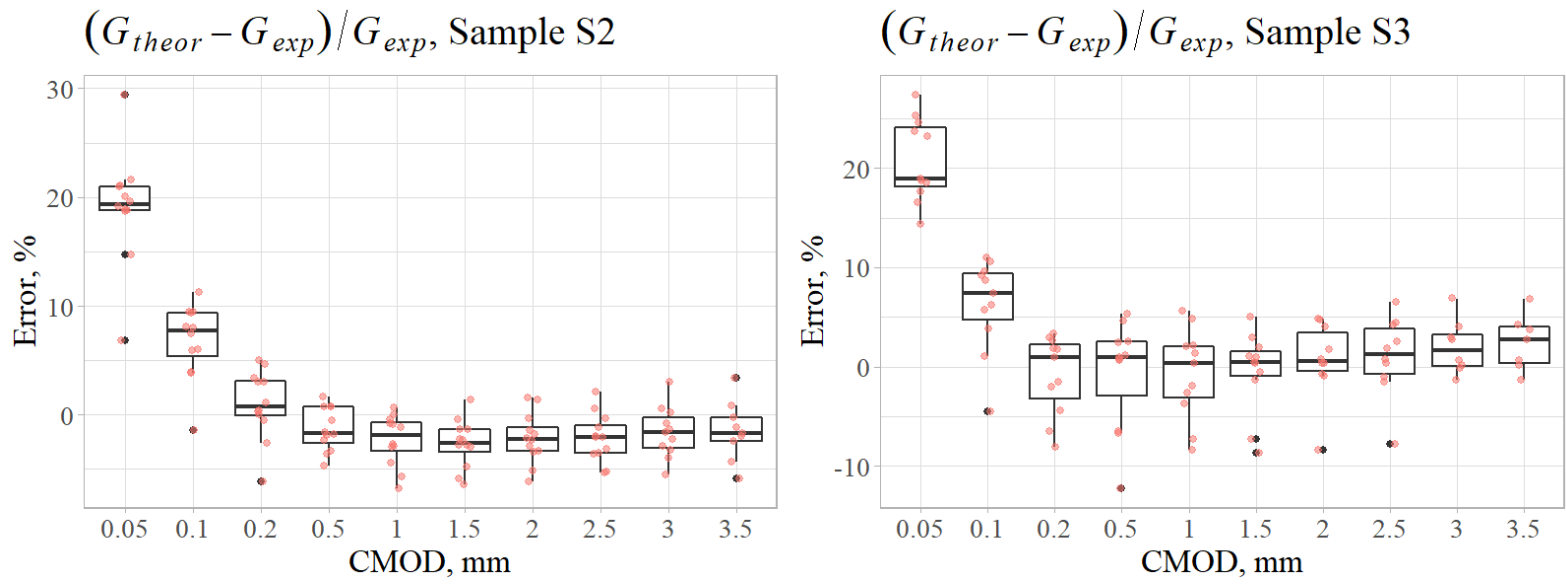

Fig. 8. Errors of theoretical $G$ values if compared to the experimental ones, represented in form of boxplots: pink dots show the actual error values 

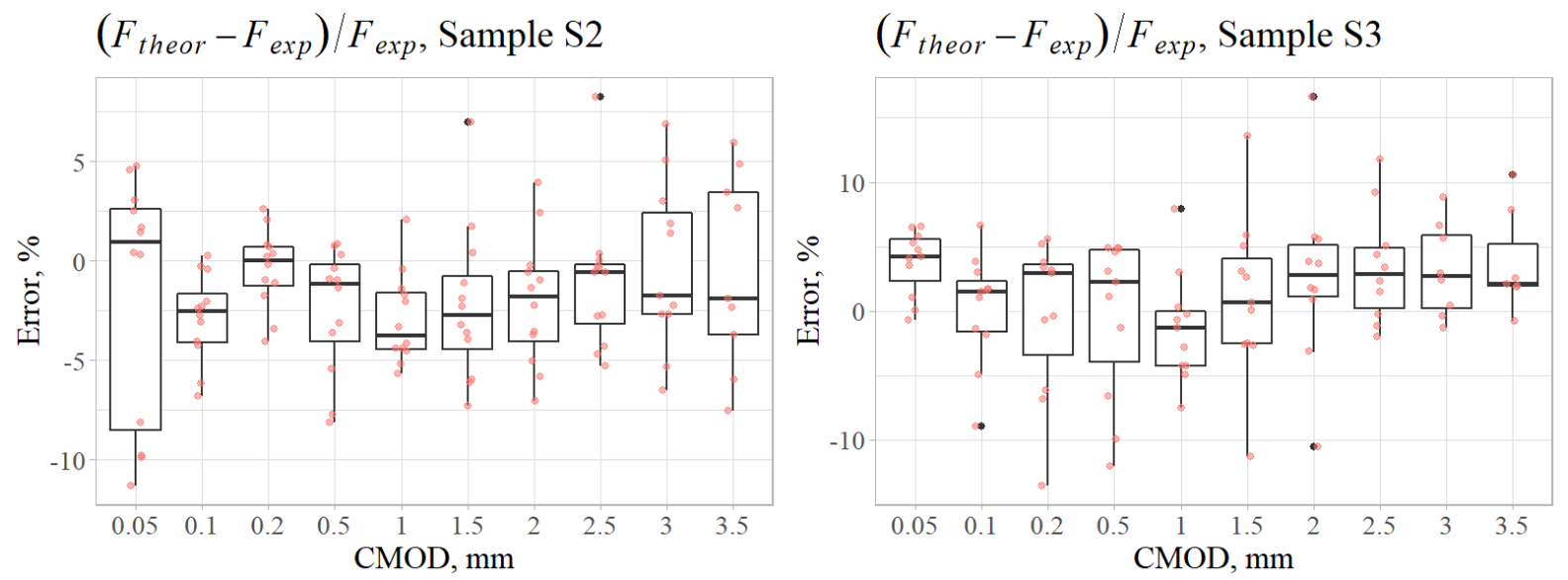

Fig. 9. Errors of theoretical $\boldsymbol{F}$ values if compared to the experimental ones, represented in form of boxplots: pink dots show the actual error values

\section{Conclusions}

1. The SFRC standard bending test results differ from the material behaviour in tension, but they can be used to derive the SFRC tension properties using mathematical formulas. The paper proposes a model to predict SFRC strength properties in tension after the limit of proportionality (LOP).

2. The evaluation of the proposed model shows $8 \%$ error at $0.1 \mathrm{~mm}$ and $\pm 2.5 \%$ error at $3.5 \mathrm{~mm}$ of the crack mouth opening distance (CMOD) in average, if the areas under the experimental and theoretical $F-C M O D$ curves or the fracture energies are compared.

3. The tension properties of SFRC obtained by the proposed model can be used as the actual material properties, or as the starting values in manual inverse analysis, if some adjustments are needed.

4. The proposed model shows rather good results for SFRC with strain softening and moderate strain hardening behaviour. However, it needs to be adjusted in case of steep increase of strength right after the LOP or other rapid changes of the residual strength.

\section{Acknowledgements}

The research is financially supported by the European Regional Development Fund. Project No. 1.1.1.2/VIAA/3/19/487, "Efficiency of fibre reinforced cement composites in structural walls".

\section{References}

[1] Marcalikova Z., Cajka R. Determination of Mechanical Properties of Fiber Reinforced Concrete for Numerical Modelling. Civil and Environmental Engineering, vol. 16, 2020, pp. 86-106.

[2] EN 14651 standard "Test method for metallic fibre concrete - Measuring the flexural tensile strength (limit of proportionality (LOP), residual)"

[3] Taerwe L., Matthys S., N. M. C. fib special activity group, fib model code for concrete structures 2010. Berlin, Germany: Ernst \& Sohn, Wiley, 2013. 402 p.

[4] Sajdlova T. ATENA Program Documentation Part 4-7, ATENA Science - GiD FRC Tutorial, Step by step guide for nonlinear analysis of fiber reinforced concrete structures with ATENA and GiD, Prague: Cervenka Consulting, 2016. 46 p.

[5] Juhasz K.P., Schaul P., Winterberg R. Influence of test methodology on the applicability of test results of fibre reinforced concrete for design. Fib Bulletin 95: "Fibre Reinforced Concrete: From Design to Structural Applications - FRC2018”, 2020. pp. 50-59.

[6] Roelfstra P.E., Wittmann F.H., Numerical method to link strain softening with failure of concrete. Fracture toughness and fracture energy of concrete. Amsterdam: Elsevier Science Publishers B.V., 1986. pp. 163-175.

[7] Sucharda O., Pajak M., Ponikiewski T., etc. Identification of mechanical and fracture properties of self-compacting concrete beams with different types of steel fibres using inverse analysis, Construction and Building Materials, vol. 138, 2017, pp. 263-275. 
[8] Sajdlova T., Pukl R., Juhasz K.P., etc. Fibre reinforced concrete constitutive laws for numerical simulation. Proceedings of 12th Central European Congress on Concrete Engineering 2017 "Modelling, Design and Codification", August 31 - September 1, 2017, Tokaj, Hungary, pp. 632-639.

[9] Novák D., Lehký D. ANN inverse analysis based on stochastic small-sample training set simulation. Engineering Applications of Artificial Intelligence, vol 19, 2006, pp. 731-740.

[10] Juhasz K.P., Modified fracture energy method for fibre reinforced concrete. Proceedings of $7^{\text {th }}$ International Conference "Fibre concrete 2013", September 12-13, 2013, Prague, Czech Republic.

[11]EN 1992-1-1 standard "Eurocode 2: Design of concrete structures - Part 1-1: General rules and rules for buildings"

[12] Kooiman A.G., Veen C., Walraven J.C. Modelling the post-cracking behaviour of steel fibre reinforced concrete for structural design purposes. HERON, vol. 45, 2000. pp. 275-307. 